\title{
Study on the effect of shikonin on CD36 expression and phagocytic ability of microglia in the isolated cerebral haemorrhage model
}

\author{
Sheng Nie ${ }^{1}$, Jikuang Zhao ${ }^{1}$, Yi Huang ${ }^{1}$, Chenhui Zhou ${ }^{1}$, Fanyong Gong ${ }^{1}$, Fei Zhao ${ }^{2}$ \\ ${ }^{1}$ Department of Neurosurgery, Ningbo First Hospital, Ningbo, Zhejiang Province, China, ${ }^{2}$ Department of Neurosurgery, \\ Yuyao People's Hospital, Yuyao, Zhejiang Province, China
}

\begin{abstract}
Aim of the study: To investigate the effects of shikonin on CD36 expression and phagocytic ability of microglia, and its protective effect on neurons and the possible mechanism within.

Material and methods: The effects of shikonin on CD36 expression and phagocytic ability of microglia were detected by Western blot method, and cerebral haemorrhage was isolated by flow cytometry in the experiment. The protective effect of neurons was observed through neuron-microglia co-culture technique. Meanwhile, the effect of hydrogen peroxide on the expression of catalase was detected, and the concentration of hydrogen peroxide was measured in the isolated cerebral haemorrhage model. The $t$ test was used to compare data between 2 groups, and one-way ANOVA was applied to multiple sets of data.

Results: Compared with the control group, the CD36 expression and phagocytic ability of microglia was increased by shikonin in the isolated cerebral haemorrhage model, while inflammatory factors such as tumour necrosis factor $\alpha$ (TNF- $\alpha$ ) and interleukin $1 \beta$ (IL-1 $\beta$ ) attenuated the effects of the drug. The amount of neuron apoptosis/necrosis was significantly reduced by the drug, while the expression of catalase in microglia was increased, but the secretion of hydrogen peroxide was decreased in the neuron-microglia co-culture system.

Conclusions: Shikonin can enhance the CD36 expression and the ability to phagocytose erythrocyte of microglia. Simultaneously, shikonin performs protective effects on neuronal cells and promotes the absorption of haematoma. Therefore, shikonin is probably an innovative medicine to treat cerebral haemorrhage.
\end{abstract}

Key words: CD36, cerebral haemorrhage, haematoma absorption, inflammation, TLR4 signalling pathway.

\section{Introduction}

Intracerebral haemorrhage (ICH), a common type of stroke, originates from non-traumatic but spontaneous bleeding in intracranial vessels, and occurs in brain parenchyma or breaks in ventricle or subarach- noid cavum. Intracerebral haemorrhage, a major cause of human death, has the highest morbidity, mortality, and disability rate, accounting for $10-15 \%$ of all brain death cases.

Toll-like receptors (TLRs) play a key role in congenital immunity and inflammatory reaction [4].

Communicating author:

Fei Zhao, Department of Neurosurgery, Yuyao People’s Hospital, Yuyao, Zhejiang Province 315410, China, phone: 86-574-62619431, fax: 86-574-62619027, e-mail: zfzhaofei12@163.com 
TLRs, comprising 13 members in the mouse family, are type I transmembrane proteins which could identify molecular patterns from different pathogens that originate from a variety of organisms such as viruses, bacteria, mycobacteria, funguses, and parasites $[1,8]$. In addition to recognizing exogenous pathogens, TLRs can also mediate host inflammatory reactions, thus leading to lesions that can be identified through lesion-associated molecular patterns $[3,6,10]$. Previous studies have shown that activated microglial cells can cause inflammatory lesions by releasing cytokines after intracerebral haemorrhage, and can inhibit microglial cell activation to improve neurological functions [7,12]. However, after the occurrence of intracranial haemorrhage, a large number of TLRs were activated; thus, the microglial cell activation was mediated.

This study explores whether shikonin is a TLR4 inhibitor, so as to promote the absorption of cerebral haemorrhage haematoma in the treatment of cerebral haemorrhage. We not only revealed the mechanism of intracerebral haemorrhage haematoma absorption, but also provided an experimental basis for the new concept of promoting haematoma absorption to treat intracerebral haemorrhage, expecting to provide a new method for the treatment of intracerebral haemorrhage.

\section{Material and methods \\ Culture of primary neurons}

A newborn 1-day-old wild-type mouse was obtained, and its neurons were separated. The stripped cerebral cortex was digested by trypsin into single cells. Neurocytes were inoculated in a $12-\mathrm{mm}$ glass coverslip on a 6-well plate, which was smeared with poly-L-lysine, and then the neurons were seeded into the neurobasal culture solution. After 5 days, the solution was replaced with serum-free B27 culture medium to inhibit the growth of neurogliocyte. The study was approved by the Yuyao People's Hospital.

\section{Culture and identification of microglial cells}

The skull of the C57/BL6 mouse was cut open under sterile conditions, the whole brain was removed and transferred to a petri dish with $3 \mathrm{ml}$ DMEM/F12, and the blood was rinsed off. The olfactory bulb, cerebellum, and meninges of the mouse were removed under a dissecting microscope, then the brain white matter and hippocampus were eliminated. Following this, the brain tissue was cut off and then put into DMEM/F12 containing $0.25 \%$ trypsin and digested at $37^{\circ} \mathrm{C}$ for $10 \mathrm{~min}$. Afterwards, DMEM/F12 + 10\% bovine serum albumin (BSA) culture solution was added to terminate the digestion. The cell suspension was gently agitated into single cells and then inoculated into a cell culture flask and placed into a $37^{\circ} \mathrm{C}$ incubator. The adherent cells were the microglial cells, which were cultured in the complete culture solution for 3 days and then digested by $0.25 \%$ trypsin. Subsequently, the single cell suspension was inoculated into 12-well plates at the density of $4 \times 10^{5} / \mathrm{ml}$. Cells were divided into 4 groups: shikonin $\left(\mathrm{C}_{16} \mathrm{H}_{16} \mathrm{O}_{5}\right.$, purity: $>98 \%$, melting point: $149^{\circ} \mathrm{C}$, boiling points: $567.4^{\circ} \mathrm{C}$, Shanghai Source Leaf Biology Company, CAS:517-89-5) $5 \mu \mathrm{M}$ group, shikonin $5 \mu \mathrm{M}+$ tumour necrosis factor $\alpha$ (TNF- $\alpha ; 100 \mathrm{ng} / \mathrm{ml}$ ) group, shikonin $5 \mu \mathrm{M}+$ interleukin (IL)-1 $\beta$ (100 $\mathrm{ng} / \mathrm{ml})$ group, and the control group (phosphate-buffered saline-PBS).

\section{Detection of CD36 expression in microglia using Western blot method}

Cells in each group were collected and rinsed with PBS twice, and to each bottle was added $400 \mu \mathrm{l}$ cell lysate plus $10 \mathrm{mmol} / \mathrm{l}$ phenylmethylsulfonyl fluoride (PMSF) $40 \mu \mathrm{l}$ in sequence. Following this, the cell culture flasks were gently shaken and then placed on ice for 10 min, making a uniform lysis. The lysis was added to the EP tube with a sterile syringe through repeated suction. The EP tube was ice-bathed for $30 \mathrm{~min}$, then centrifuged for $15 \mathrm{~min}$ at $12,000 \mathrm{~g}$. The supernatant was decanted into a new EP tube, then the protein concentration was quantitated with a microplate reader using protein-labelled BC method. Subsequently, $20 \mu$ l protein sample $6 \times$ buffer was added into each tube at every $100 \mu \mathrm{l}$ and boiled for $5 \mathrm{~min}$, mixed, and then stored at $-80^{\circ} \mathrm{C}$. Following this, the protein sample was separated through 12\% SDS-PAGE electrophoresis, and the separated protein bands were transferred to PVDF membrane by wet method, and then sealed for $1 \mathrm{~h}$ at room temperature. The primary antibody CD36 at concentration 1 : 1000 was added to the protein bands and incubated overnight at $4^{\circ} \mathrm{C}$, then the cells were washed with phosphate-buffered saline with Tween ${ }^{\circledR} 20$ (PBST) 3 times. Afterwards, the second- 
ary antibody at a concentration of $1: 1000$ was added and incubated for $1 \mathrm{~h}$, and then rinsed with PBST 3 times. Finally, the protein expression in each group was determined by chemiluminescence method for further colour development and fixation.

\section{Detection of CD36 expression in microglia by flow cytometry}

Primary microglial cells were added to each group, and carboxyfluorescein succinimidyl ester (CFSE)-labelled red blood cells (RBCs) were added to the microglial cells and then incubated at $37^{\circ} \mathrm{C}$ for $12 \mathrm{~h}$. After the collection, the microglial cells were stained by anti-mouse CD36-PerCP-eFluor ${ }^{\circledR} 710$ and CD11b antibody-PE, and the change of CD36 expression was detected by flow cytometry.

\section{Detecting the phagocytosis ability of microglia using flow cytometry}

Primary microglial cells were added to each group, simultaneously, CFSE-labelled RBCs were added, and they were incubated at $37^{\circ} \mathrm{C}$ for $12 \mathrm{~h}$. Microglial cells were dyed by CD11B-PE and collected. Finally, the percentage of microglia involved in erythrocyte phagocytosis, the average fluorescence intensity of CFSE in PE + CFSE + microglia, and the numbers of RBCs that were not phagocytosed in the supernatant, were all calculated.

\section{Detection of neurotoxicity and drug protective effects through co-culture of neuron-microglia}

In order to evaluate the microglia-induced neurotoxicity and the protective effects of shikonin, C57 neonatal rat neuron cells at the density of $1 \times 10^{5} / \mathrm{ml}$ were inoculated into a poly-L-lysine smeared $12-\mathrm{mm}$ glass coverslip on a 24-well plate. Subsequently, microglia at the density of $1 \times 10^{5} / \mathrm{ml}$ in the upper part of the neuron cells was inoculated into Transwell and cultured in DMEM/F12 containing $10 \%$ foetal calf serum at $37^{\circ} \mathrm{C}$ with $5 \% \mathrm{CO}_{2}$ for $48 \mathrm{~h}$. Erythrocytes were added to the Transwell after $2 \mathrm{~h}$ of $5 \mu \mathrm{M}$ shikonin or PBS stimulation, and the neurons were fixed and stained after $12 \mathrm{~h}$. The cell nuclei were first dyed by anti-mouse neuron $\beta$ III-tubulin, then by Alexa Fluor 488 goat-anti-mouse IgG and Hoechst. Finally, the neuron cell image formation was presented by laser scanning confocal microscopy to determine the survival rate of neuron cells.

\section{Detection of hydrogen peroxide and catalase}

The microglia suspension at a density of $1 \times 10^{5} / \mathrm{ml}$ was inoculated into 6-well plates, and erythrocytes were added; simultaneously, $5 \mu \mathrm{M}$ of shikonin or PBS were added, respectively. The supernatant was collected after $12 \mathrm{~h}$ of incubation, and the concentration of hydrogen peroxide as well as the activity of catalase were determined according to the kit instructions.

\section{Statistical approach}

All data are presented as mean \pm standard deviation (mean $\pm \mathrm{SD}$ ). The $t$ test was used to compare data between 2 groups, while one-way ANOVA was applied to multiple sets of data $(>2)$. $P<0.05$ was considered statistically significant. All data were analysed and processed by Graphpad Prism 5.0 (Graphpad Software, San Diego, CA).

\section{Results and discussion \\ CD36 expression in microglia as promoted by shikonin}

The results showed that, compared with the PBS control group, the CD36 expression in the shikonin group significantly increased, was distinctly enhanced in the shikonin + IL-1 $\beta$ group, and was slightly intensified in the shikonin + TNF- $\alpha$ group. However, the CD36 expressions in the shikonin + IL-1 $\beta$ group and the shikonin + TNF- $\alpha$ group were decreased in contrast to the shikonin group, suggesting that IL-1 $\beta$ and TNF- $\alpha$ can attenuate the promoting effects of shikonin on CD36 expressions (Fig. 1).

\section{Detection of CD36 expression change in microglia by flow cytometry}

The experiment clearly demonstrated that the result detected by flow cytometry is in accordance with the Western blot result (Fig. 2).

\section{Detection of microglia phagocytic ability change by flow cytometry}

The results indicated that the percentage of microglia involved in erythrocyte phagocytosis in the shikonin group was remarkably higher than that in the PBS group, the average fluorescence intensity of CFSE was higher than that in the PBS group, but the 


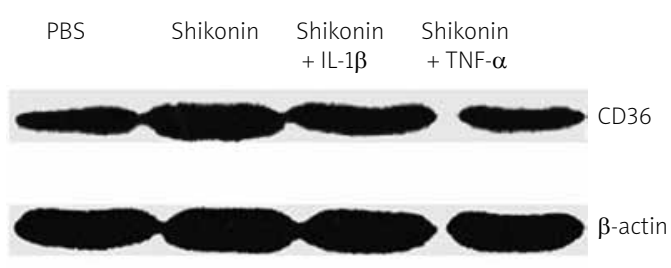

Fig. 1. CD36 expression in microglia promoted by shikonin $(n=6)$.

RBC that had not been phagocytosed was significantly reduced. However, this phenomenon can be distinctly attenuated by IL-1 $\beta$ and TNF- $\alpha$, which is reflected in the result that the percentage of microglia involved in erythrocyte phagocytosis is lower in both the shikonin $+\mathrm{IL}-1 \beta$ group and the shikonin + TNF- $\alpha$ group than that in the shikonin group. In addition, the average fluorescence intensity of CFSE in microglia which had phagocytosed erythrocyte is evidently lower than that in the shikonin group, and the RBC which had not been phagocytosed also significantly increased. It can be concluded that the ability of phagocytosis of erythrocytes by microglia increased in the shikonin group, but the effect could be weakened by IL-1 $\beta$ and TNF- $\alpha$ (Figs. 3-5).

\section{Effects of shikonin on neuronal protection}

The results showed that the number of neurons that survived in the shikonin group evidently increased in comparison with the PBS group, suggesting that shikonin plays a protective role during the process of cerebral haemorrhage (Fig. 6).

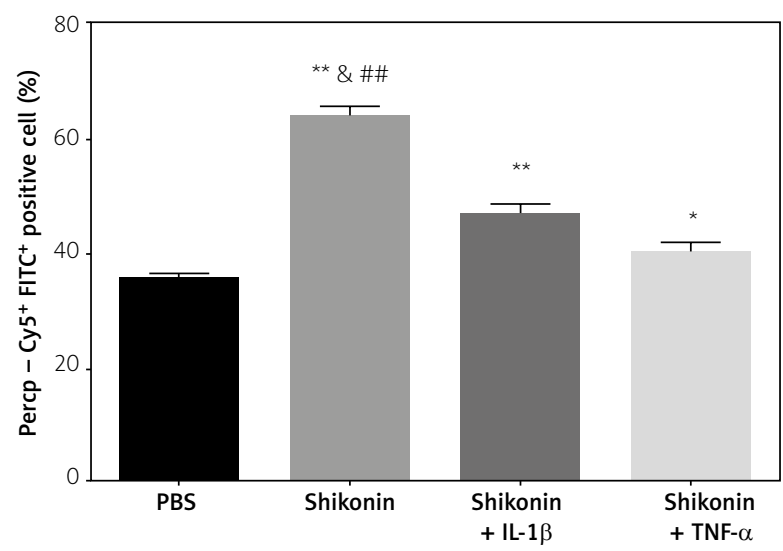

Fig. 3. Effect of shikonin on red blood cells (RBCs) phagocytosis by microglia $(n=6) .{ }^{* *} p<0.01$ vs. PBS, ${ }^{*} p<0.05$ vs. PBS, $\&_{p}<0.05$ vs. shikonin + IL-1 $\beta,{ }^{\# \#} p<0.01$ vs. shikonin + TNF- $\alpha$.

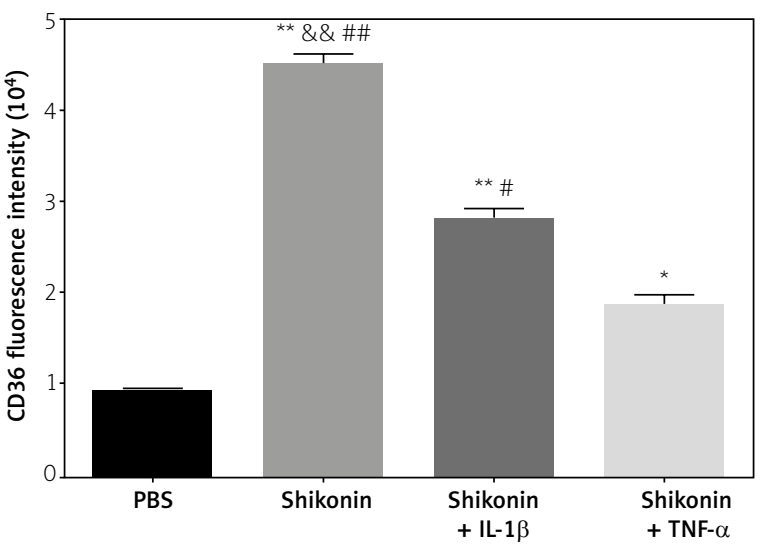

Fig. 2. Effects of shikonin on CD36 expression in microglia $(n=6) .{ }^{* *} p<0.01$ vs. PBS, ${ }^{*} p<0.05$ vs. PBS, $\& \& p<0.01$ vs. shikonin $+\mathrm{IL}-1 \beta$, ${ }^{\# \#} p<0.01$ vs. shikonin + TNF- $\alpha,{ }^{\#} p<0.05$ vs. shikonin + TNF- $\alpha$.

\section{Effects of shikonin on catalase expression and hydrogen peroxide secretion in microglia}

Although shikonin has a neuro-protective effect, the potential mechanism remains unclear. In the course of cerebral haemorrhage, a good deal of reactive oxygen was produced, thus leading to brain damage. In a bid to fight with the oxidative stress caused by cerebral haemorrhage, cells secrete certain self-defendant anti-oxidases, like catalase, which plays an important role in the process of eliminating reactive oxygen by decomposing hydrogen peroxide. In addition to producing a large number of inflammatory factors during the course of microglia activation

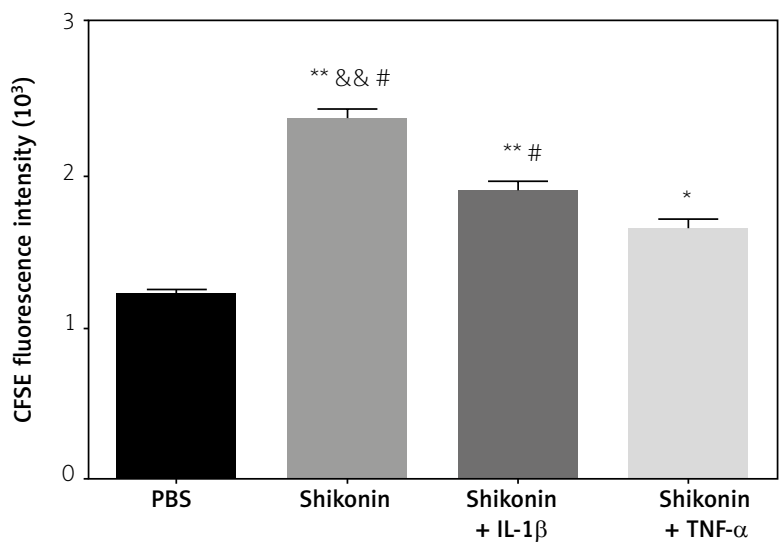

Fig. 4. Effects of shikonin on fluorescence intensity of CFSE in microglia $(n=6) .{ }^{* *} p<0.01$ vs. PBS, ${ }^{*} p<0.05$ vs. PBS, \&\& $p<0.01$ vs. shikonin + IL-1 $\beta$, ${ }^{\# \#} p<0.01$ vs. shikonin + TNF- $\alpha$, ${ }^{\#} p<0.05$ vs. shiko$\operatorname{nin}+$ TNF- $\alpha$. 


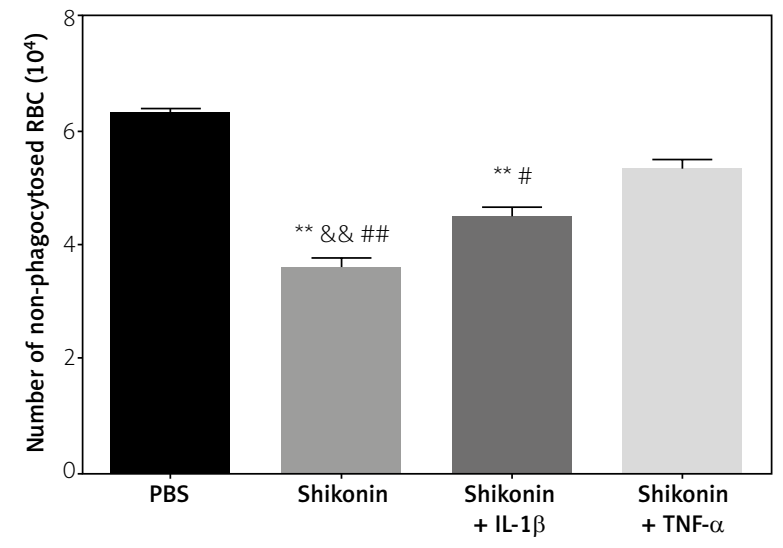

Fig. 5. Effect of shikonin on amounts of red blood cell (RBC) that had not been phagocytosed in the supernatant $(n=6) .{ }^{* *} p<0.01$ vs. PBS, $\& \& p<0.01$ vs. shikonin + IL-1 $\beta$, ${ }^{\# \#} p<0.01$ vs. shikonin + TNF- $\alpha,{ }^{*} p<0.05$ vs. shikonin + TNF- $\alpha$.

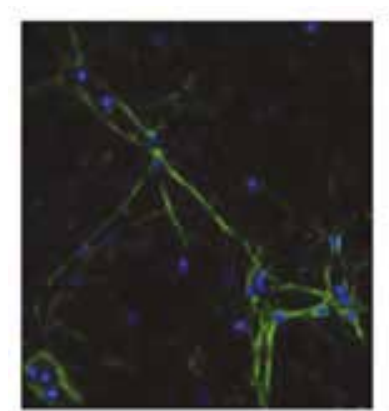

PBS

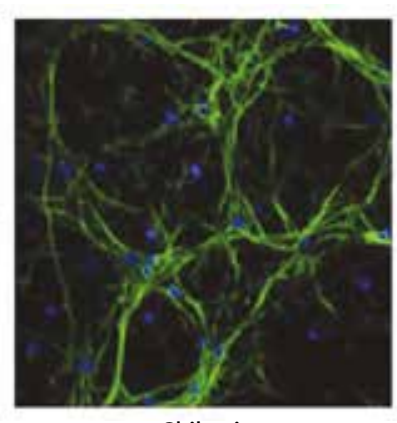

Shikonin

after cerebral haemorrhage, a lot of reactive oxygen was generated, resulting in damage to the microglial cells and surrounding neurocytes. The present study revealed that the expression of hydrogen peroxide in the supernatant of cell culture medium was clearly reduced, indicating that the neuro-protective effect of shikonin may increase the survival rate of neurocytes by accelerating the expression of catalase in microglia, thus reducing hydrogen peroxide and oxidative stress (Figs. 7 and 8).

\section{Discussion}

Inflammation is a critical factor in secondary brain injury caused by cerebral haemorrhage. And inflammation response is caused by a variety of stimuli after $\mathrm{ICH}$. Haematoma components trigger the inflammatory signal by activating microglia, which were followed by the release of early inflammatory

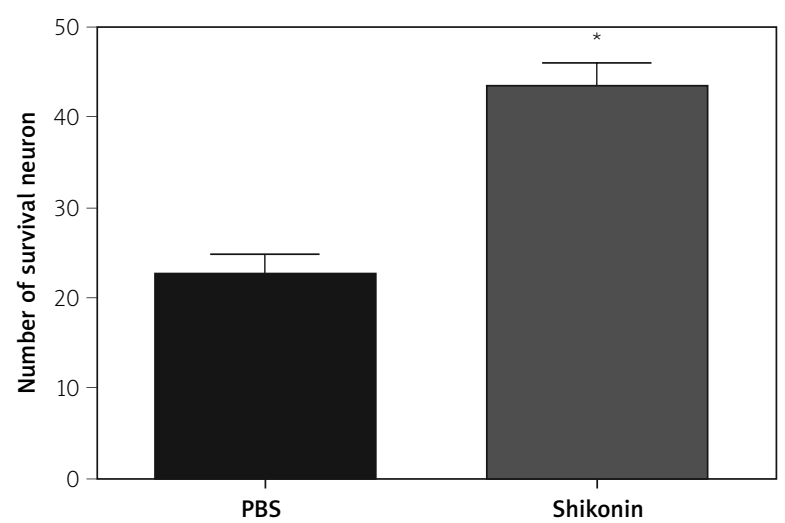

Fig. 6. Effects of shikonin on neuronal protection $(n=6)$.

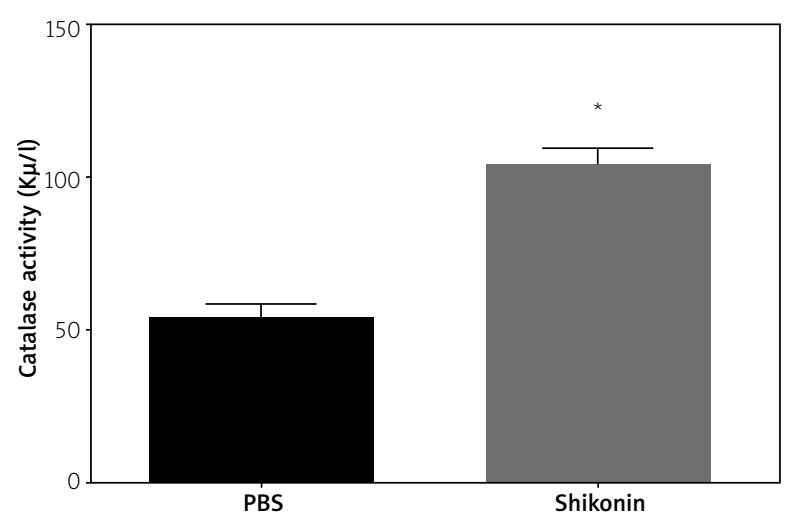

Fig. 7. Effects of shikonin on catalase activity $(n=6) .{ }^{*} p<0.01$ vs. PBS.

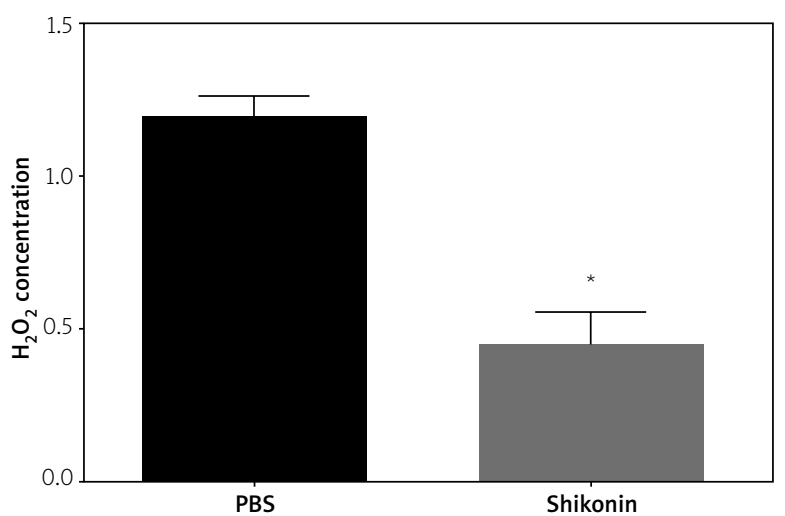

Fig. 8. Effects of shikonin on hydrogen peroxide release $(n=6) .{ }^{*} p<0.01$ vs. PBS. 
factors and chemotactic factors, attracting ambient inflammatory cells to infiltrate [5]. The released haemoglobin $(\mathrm{Hb})$, haeme, and iron after erythrocyte splitting aggravate $\mathrm{ICH}$-induced inflammatory damage. And the release of hazard-related molecular patterns from damaged or dead cells, such as high mobility group protein 1 , can cause inflammation in the late phase of cerebral haemorrhage. Preclinical studies have found that the inflammatory signalling pathways are involved in microglia activation, leukocyte infiltration, TLR activation, and hazard-related molecular pattern adjustment after cerebral haemorrhage $[13,15]$.

CD36 expresses itself in brain neurocytes, astrocytes, and microglia, and with different corresponding functions. In astrocytes, the role of CD36 is to promote the proliferation of astrocytes, and to participate in the formation of glial scar generated by mediating stroke. If the expression of CD36 were inhibited, the formation of intermediate filament after injury can be reduced, thereby decreasing the formation of glial scar [2]. In some neuron cells in the cerebrum, CD36 functions in the brain nucleus information loop and is involved in pheromone response, reproductive behaviour, and food preferences [11].

Cultured microglia start the phagocytosis to erythrocytes, the main components of haematoma through CD36, which leads to the secretion of inflammatory factors such as TNF- $\alpha$ and IL-1 $\beta$, as well as the increase of inducible nitric oxide synthase (iNOS) and matrix metalloproteinase (MMP-9) expressions $[9,14]$. Cultured microglia began to expand with antennae within a few minutes of exposure to erythrocytes, and in a few hours RBC was attached to microglia. This process was accompanied by the increase of CD36 expression. It has been found that microglia displayed amoebic-like changes, followed by the increase of $\mathrm{CD} 36$ expression in the animal cerebral haemorrhage model [14]. Therefore, in the course of cerebral haemorrhage, erythrocytes induce the activation of microglia through CD36.

The inflammatory factors such as TNF- $\alpha$ and IL-1 $\beta$ produced by TLR4 signalling pathway activation can inhibit the expression of CD36. If the TLR4 signal were inhibited, there would be less TNF- $\alpha$ and IL-1 $\beta$ preoduced, hence alleviating the inhibitory effects on CD36 expression. The present study discovered that the use of shikonin interdicts the activation of TLR4 signalling pathway and decreases the production of both TNF- $\alpha$ and IL- $1 \beta$, which inevitably increases the expression of CD36. Moreover, this can be proven by the results of using shikonin $+\mathrm{IL}-1 \beta$ and shikonin + TNF- $\alpha$ combined. The use of TNF- $\alpha$ and IL-1 $1 \beta$ offsets the decrease of secretion resulted from shikonin interdicting TLR4 signalling pathway, and inhibits the expression of CD36, which proved otherwise that shikonin promotes CD36 expression mainly through inhibiting TLR4 signalling pathway. Also, the phagocytosis of microglia towards erythrocyte was enhanced after the administration of shikonin, which is related to the increase of CD36 expression. However, the employment of TNF- $\alpha$ and IL-1 $\beta$ combined weakened the phagocytic ability of microglia, which once again confirmed that shikonin can affect the expression of CD36 through inhibiting TLR4 signalling pathway. After adding erythrocytes into the co-culture system of microglia and neuron cells, the catalase in the shikonin group was significantly increased, while the hydrogen peroxide was conspicuously decreased, along with increased survival of neuron cells. This suggested that shikonin can not only reduce the production of inflammatory factors and lessen nerve injuries by restraining TLR4 signal, but also decrease the oxidative stress caused by cerebral haemorrhage. Furthermore, shikonin can also increase the survival of neurons by increasing the expression of catalase. However, the mechanism of shikonin increasing the expression of catalase in cells is still unclear, which requires further studies.

\section{Ethics approval and consent to participate}

The study was approved by the Yuyao People's Hospital. A newborn 1-day-old wild-type mouse was obtained and its neurons were separated.

\section{Funding}

This study was supported by the Medicine and Health Science and Technology Projects of Zhejiang Province (2017KY586, 2017KY588).

\section{Disclosure}

The authors report no conflict of interest.

\section{References}

1. Barichello T, Generoso JS, Goularte JA, Collodel A, Pitcher MR, Simoes LR, Quevedo J, Dal-Pizzol F. Does infection-induced immune activation contribute to dementia? Aging Dis 2015; 6: 342-348. 
2. Deng C, Ren GY, Sun AN, Luo XP, Wang ZT, Dou W. The regulatory effect of cardamonin on TLR4/MyD88/NF-KB/Inos pathway. Chin Pharmacol Bull 2016; 32: 779-783.

3. Gao W, Zhao Z, Yu G, Zhou Z, Zhou Y, Hu T, Jiang R, Zhang J. VEGI attenuates the inflammatory injury and disruption of bloodbrain barrier partly by suppressing the TLR4/NF-kappaB signaling pathway in experimental traumatic brain injury. Brain Res 2015; 1622: 230-239.

4. Guerrero AT, Pinto LG, Cunha FQ, Ferreira SH, Alves-Filho JC, Verri WA Jr, Cunha TM. Cunha. Mechanisms underlying the hyperalgesic responses triggered by joint activation of TLR4. Pharmacol Rep 2016; 68: 1293-1300.

5. He Y, Yue Y, Zheng X, Zhang K, Chen S, Du Z. Curcumin, inflammation, and chronic diseases: how are they linked? Molecules 2015; 20: 9183-9213.

6. Lee JY, Lee JD, Phipps S, Noakes PG, Woodruff TM. Absence of toll-like receptor 4 (TLR4) extends survival in the hSOD1 G93A mouse model of amyotrophic lateral sclerosis. J Neuroinflammation 2015; 12: 90.

7. Liang DY, Liu F, Chen JX, He XL, Zhou YL, Ge BX, Luo LJ. Porphyromonas gingivalis infected macrophages upregulate CD36 expression via ERK/NF- B pathway. Cell Signal 2016; 28: 12921303.

8. Mahmood K, Zia KM, Zuber M, Salman M, Anjum MN. Recent developments in curcumin and curcumin based polymeric materials for biomedical applications: a review. Int J Biol Macromol 2015; 81: 877-890.

9. Mohammad Hosseini A, Majidi J, Baradaran B, Yousefi M. Tolllike receptors in the pathogenesis of autoimmune diseases. Adv Pharm Bull 2015; 5: 605-614.

10. Ni H, Jin W, Zhu T, Wang J, Yuan B, Jiang J, Liang W, Ma Z. Curcumin modulates TLR4/NF-kappaB inflammatory signaling pathway following traumatic spinal cord injury in rats. J Spinal Cord Med 2015; 38: 199-206.

11. Saifudeen I, Subhadra L, Konnottil R, Nair RR. Metabolic modulation by medium chain triglycerides reduces oxidative stress and ameliorates CD36 mediated cardiac remodeling in spontaneously hypertensive rat in initial and established stages of hypertrophy. I Card Fail 2016; 23: 240-251.

12. Shi YW, Zhang XC, Chen C, Tang M, Wang ZW, Liang XM, Ding F, Wang CP. Schisantherin A attenuates ischemia/reperfusion-induced neuronal injury in rats via regulation of TLR4 and C5aR1 signaling pathways. Brain Behav Immun 2017; 66: 244-256.

13. Sordillo PP, Helson L. Curcumin suppression of cytokine release and cytokine storm. A potential therapy for patients with Ebola and other severe viral infections. In Vivo 2015; 29: 1-4.

14. Wang J, Wang H, Zhu R, Liu Q, Fei J, Wang S. Anti-inflammatory activity of curcumin-loaded solid lipid nanoparticles in IL-1beta transgenic mice subjected to the lipopolysaccharide-induced sepsis. Biomaterials 2015; 53: 475-483.

15. Xun W, Shi L, Zhou H, Hou G, Cao T, Zhao C. Effects of curcumin on growth performance, jejunal mucosal membrane integrity, morphology and immune status in weaned piglets challenged with enterotoxigenic Escherichia coli. Int Immunopharmacol 2015; 27: 46-52. 